\title{
Educational exercise program affects to physical fitness and gross motor function differently in the severity of autism spectrum disorder
}

\author{
Jieun Yu', Yong-Seok Jee ${ }^{2, *}$ \\ 'Department of Physical Education, Korea University, Seoul, Korea \\ ${ }^{2}$ Research Institute of Sports and Industry Science (RISIS), Hanseo University, Seosan, Korea
}

This study investigated the effects of participating in an educational exercise program on physical fitness and gross motor function (GMF) in adults with varying degrees of autistic spectrum disorder (ASD). The subjects consisted of 35 voluntary male participants between 20 and 29 years of age who were allocated to one of two groups: mild ASD $(n=17)$ group and severe ASD ( $n=18)$ group. All selected tests for physical fitness, including body composition and GMF, have been used in previous studies. The results were as follows: first, with the exception of the basal metabolic rate, there were significant differences in the interaction of all other body composition variables. Second, there were significant differences in the interaction of almost all physical fitness variables, except for muscle strength. Finally, although there were significant differences in the interaction of all variables, except the locomotion skill for hopping, there were significant differences in the interaction of all variables of object control skill. Specifically, although the $\Delta \%$ in the sum of locomotion skill in mild ASD group increased $\sim 19.81 \%$, that of severe ASD group decreased $\sim 4.78 \%$. The $\Delta \%$ in the sum of object control skill in mild ASD group improved $29.96 \%$, while that of severe ASD group reduced $\sim 15.2 \%$. In conclusion, it is thought that these results are due to the better understanding of educational exercise and better performance of educational exercise in adults with mild ASD compared to adults with severe ASD.

Keywords: Autistic spectrum disorder, Physical fitness, Gross motor function

\section{INTRODUCTION}

The World Health Organization refers to the concept of disability as a loss of body structure or function, abnormality or impairment that implies restrictions on the activities of individuals in daily life (Barbotte et al., 2001). The reason for this is that the definition of disabled person is defined differently according to the law, and the concept of disability differs in understanding and interest according to social, political and economic conditions (Howlin and Moss, 2012). Recently, autistic spectrum disorder (ASD) is attracting a greater amount of interest. A 2007 survey by the Ministry of Health and Welfare reported that children with ASD accounted for $12.5 \%$ among the total number of people with disabilities. ASD is a mental illness that affects more than 35 mil- lion people worldwide. It is an unidentified disease that continues to affect an increasing number of people, but has no preventive measures or treatments (Brugha et al., 2011). Moreover, the prevalence of ASD is frequently reported to be one out of 110 people. There is no cure since no exact cause is known. Instead of relying on medication, many experts argue that alternative therapies should be designed to increase the effectiveness of treatment. Currently, special education programs and treatment for children with ASD are being implemented in various fields. In particular, special education, rehabilitation programs, cognitive behavioral therapy, and various art therapies are provided (Baird et al., 2006).

An active educational exercise program can improve the health of children with disabilities and promote psychological stability. Many researchers have reported that participating in physical ac-
${ }^{*}$ Corresponding author: Yong-Seok Jee (iD https://orcid.org/0000-0001-6797-0843 Research Institute of Sports and Industry Science (RISIS), Hanseo University, 46 Hanseo 1-ro, Haemi-myeon, Seosan 31962, Korea

E-mail: jeeys@hanseo.ac.kr

Received: September 1, 2020 / Accepted: September 22, 2020
This is an Open Access article distributed under the terms of the Creative Commons Attribution Non-Commercial License (https://creativecommons.org/licenses/by-nc/4.0/) which permits unrestricted non-commercial use, distribution, and reproduction in any medium, provided the original work is properly cited. 
tivity during educational exercise programs can provide psychophysiological benefits for children or adults with ASD (Ahn and Fedewa, 2011; Janssen and LeBlanc, 2010; Lang et al., 2010; Latorre Román et al., 2015). In addition to improving psychophysiological development, the physical activity also enhances the ability of the large and small muscles to function and protect the body (Verschuren et al., 2016). In other words, children with ASD not only engage in physical movement for improved physical function, but also gains mental satisfaction.

In Korea, educational exercise programs for children with ASD are available at certain institutions, but it is unknown whether there are differentiated programs according to the level of disability. Also, most educational exercise programs, as well as exercise programs for people with disabilities, are primarily focused only on children. This has led to a lack of research pertaining to adults with ASD. In particular, if educational exercise programs in Korea are differentiated according to disability levels, more meaningful changes may result. Therefore, in order to solve the problem, this study analyzed the educational exercise program performed by adults with ASD and identified the differences in physical fitness and gross motor function (GMF) according to their severity of ASD.

\section{MATERIALS AND METHODS}

\section{Participants}

Male adults between 20 to 29 years of age voluntarily participated in this study. Inclusion criteria consisted of having ASD and not having exercised regularly for over 6 months. Based on this criteria, 40 participants were screened and determined to be eligible for the study. However, five participants declined to participate. Finally, 35 participants took part in the study. The participants were divided into mild ASD group ( $\mathrm{n}=17)$ and severe ASD group $(\mathrm{n}=18)$ depending on the symptom severity. Those with physical impairment or visual or hearing impairment were excluded. Furthermore, participants who were unable to be assessed or required to quit the study were excluded. Those who received any kind of medical treatment that is known to have an effect on physical condition or had any kind of major surgery for the past year prior to the beginning of this study were excluded. Additional reasons for exclusion included having a history of cerebrovascular or coronary arterial disease, uncontrolled hypertension, or impairment of a major organ. Comprehensive physical characteristics are shown in Table 1.
Table 1. Physical characteristics of the participants

\begin{tabular}{lcccc}
\hline \multirow{2}{*}{ Variable } & \multicolumn{2}{c}{ Group } & \multirow{2}{*}{$Z$} & $P$-value \\
\cline { 2 - 3 } & Mild ASD $(\mathrm{n}=17)$ & Severe ASD $(\mathrm{n}=18)$ & & \\
\hline Age $(\mathrm{yr})$ & $22.82 \pm 2.70$ & $22.83 \pm 2.81$ & -0.151 & 0.883 \\
Height $(\mathrm{cm})$ & $174.01 \pm 7.16$ & $173.39 \pm 8.56$ & -0.017 & 0.987 \\
Weight $(\mathrm{kg})$ & $73.02 \pm 12.63$ & $72.09 \pm 10.32$ & -0.066 & 0.961 \\
\hline
\end{tabular}

Values are presented as mean \pm standard deviation.

ASD, autism spectrum disorder.

\section{Experimental protocol}

This was a double-blind, controlled trial that was carried out in a research center from February 17, 2020 to May 8, 2020. The participants and their parents were encouraged and motivated to complete all of the tests. Advertisements were used to recruit the participants and an informed consent form was signed prior to enrollment. The parents of the participants also agreed to their family participating in this study by signing an informed consent form. This study was completed in compliance with the 2013 version of The Declaration of Helsinki and approved (2-7001793AB-N-012019113HR) by the Institutional Review Board.

\section{Measurement of body composition}

The bioelectrical impedance analysis method was used to measure the height and weight of the participants with an IOI 353 Model (Jawon Medical, Seoul, Korea), which is a composition analyzer and segmental impedance device that has stainless steel interfaces for the electrodes. The participants stood upright by placing their feet on the foot electrodes without gripping the handles. Analysis of height and weight was measured before dinner and after voiding (Cha et al., 2014; Kim and Jee, 2020). The variables of body composition in this study were body weight, body mass index (BMI), muscle mass, fat mass, and basal metabolic rate (BMR).

\section{Measurement of cardiopulmonary fitness}

Before the test, an expert described the procedures and had the subjects do warm-ups. A $15-\mathrm{m}$ shuttle run test was used to measure cardiopulmonary endurance. This test is a commonly used aerobic fitness test, which is also known as a beep or bleep test (Yoon et al., 2019). To complete this test, the following equipment were used: a flat, nonslip surface, $15 \mathrm{~m}$ of measuring tape, marking cones, a music player, a beep test audio, and recording sheets. Participants continuously ran between two lines that were $15 \mathrm{~m}$ apart according to the recorded beeps. The participants stood behind one of the lines facing the other line, and started running when 
Table 2. Bleep test's table for cardiopulmonary endurance fitness

\begin{tabular}{|c|c|c|c|c|c|c|c|c|c|c|c|c|c|c|c|c|}
\hline Level & 1 & 2 & 3 & 4 & 5 & 6 & 7 & 8 & 9 & 10 & 11 & 12 & 13 & 14 & 15 & 16 \\
\hline Shuttles & 7 & 8 & 8 & 8 & 9 & 9 & 10 & 10 & 10 & 11 & 11 & 12 & 12 & 12 & 13 & 13 \\
\hline Time/shuttle (sec) & 6.83 & 6.36 & 6.11 & 5.75 & 5.43 & 5.13 & 4.97 & 4.78 & 4.59 & 4.39 & 4.16 & 3.92 & 3.85 & 3.76 & 3.59 & 3.47 \\
\hline Total distance (m) & 105 & 225 & 345 & 465 & 600 & 735 & 885 & 1,035 & 1,185 & 1,350 & 1,515 & 1,695 & 1,875 & 2,070 & 2,265 & 2,460 \\
\hline Total time (min:sec) & $0: 48$ & 1:39 & $2: 28$ & $3: 14$ & 4:02 & $4: 49$ & $5: 38$ & $6: 26$ & $7: 11$ & 8:00 & $8: 56$ & $9: 33$ & $10: 19$ & 11:08 & $11: 55$ & $12: 40$ \\
\hline
\end{tabular}

the signal was given by the recorded beep. The speed at the start was slow. The participants continued to run between the two lines according to the pace of the recorded beeps. Approximately $1 \mathrm{~min}$ later, the intervals between each beep became shorter, thereby increasing the pace. Each subsequent minute (level), the intervals continue to become shorter. If the participant reached the line before the following beep, they waited for it before running again. In the case that the participant did not reach the line before the following beep, a warning was given, but they continued to run and tried to catch up to the pace of the beeps. After a participant failed to reach within $2 \mathrm{~m}$ of the line for the first time, a warning was given. After the second warning, the participant was eliminated. The final score was calculated by their highest level and the total number of shuttles completed before being eliminated. The last completed level was recorded by the supervisor. The standard Australian bleep test is provided in Table 2, which provides a list of scores for comparative reference on what to expect for adults.

\section{Measurement of flexibility}

To measure flexibility, a sit and reach flexion test was administered. The equipment used included a test platform with a perpendicularly attached ruler (TKK1859, Takei Inc., Tokyo, Japan). In a seated position, the participants placed their heels against the test platform with their both feet approximately $5 \mathrm{~cm}$ apart. After taking a deep breath, the participants reached forward as they exhaled and extended their fingertips to the furthest possible point without bouncing their waists. They kept their knees fully extended and their head between their arms. The greatest distance after two attempts was recorded (Kim and Jee, 2020).

\section{Measurement of muscle strength}

Grip strengths of both hands were measured to assess muscle strength. It was measured with a hand dynamometer (preferably the Jamar Hand Dynamometer \#5030J1, Sammons Preston Rolyan, Bolingbrook, IL, USA) in a seated position. Participants sat in a seat with an armrest and had their shoulders positioned at $0^{\circ}$ flexion, elbows at $90^{\circ}$ flexion, and wrists in the vertical position between supination and pronation. Participants held the hand dy- namometer with the middle phalanges resting in the middle. The hand dynamometer was squeezed 3 times with maximum force with 1 minute of recovery between attempts. This had to be done for both hands in case handedness was not self-evident (Oppewal et al., 2013). The maximum produced force out of the six attempts was used as the test result and calculated to the nearest $\mathrm{kg}$. Test results were accepted only if the instructor conducting the test was convinced that maximum effort was applied. To verify whether maximum effort was being used, the instructor observed the consistency of the attempts, facial expressions, the contraction of muscles in the arm and hand, and the tension in the phalanges.

\section{Measurement of muscle power}

To assess muscle power, a standing long jump was used. In a standing long jump, the participant begins at the starting line. Using their entire bodies, they jump as far as possible and the distance between the starting line and the landing point is measured. The test instructors performed long jump exercises and demonstrated how to jump in place. For this task, both groups of participants initially lacked comprehension. Later, some participants jumped beyond the target footstep, while others did not. Regardless, the distance was recorded. After landing, measurement errors can be caused from falling back or running forward. In preparation for this, the two measurements were quickly recorded quickly by two assistants. The maximum distance was recorded between a total of two measurements.

\section{Measurement of GMF}

This study used the GMF scale-II (Cha et al., 2020). This allows the instructor to accurately and quickly measure any delays in underlying motion patterns of the participants compared to their peers. A total of twelve major muscle movement patterns were separated into locomotion skills and object control skills. The locomotion section included running, hopping, galloping, leaping, sliding, and horizontal jumping. The object control included striking a baseball, dribbling a basketball, catching a basketball, kicking a soccer ball, overhand throwing a tennis ball, and underhand rolling a softball. After analyzing the participants' basic mo- 
Table 3. Educational play program

\begin{tabular}{llcc}
\hline Item & \multicolumn{1}{c}{ Play and activity programs } & Time (min) & Week \\
\hline Warm-ups & Stretching & 5 & $1-12$ \\
Game & Catching a tail & 15 & $1-4$ \\
& Playing tug-of-war & 15 & $1-4$ \\
Floorball & Play for passing and receiving a ball & 15 & $1-4$ \\
& Passing a ball (slapper, air pass) & 15 & $1-4$ \\
& Mini-short game & 15 & $5-8$ \\
Basketball & Dribble (a hand dribble then both hands & 15 & $5-8$ \\
& $\quad$ dribble) & & \\
& Pass (chest, overhand, one-hand, bound) & 15 & $5-8$ \\
& Shot (middle, layup, pass and shoot) & 15 & $5-8$ \\
Inline skating & Skating adaptation (wearing, falling and & 10 & $5-8$ \\
& $\quad$ standing) & & \\
& Walking with an expert & 5 & $5-8$ \\
& Pushing (kneeling, left or right foot push) & 15 & $9-12$ \\
& Turn (return target, turn left/ right/ front/ & 15 & $9-12$ \\
& back) & & \\
Jumping paly & Sprint, jump over obstacles, running jump & 20 & $9-12$ \\
& High jump, triple jump & 20 & $9-12$ \\
Cool-down & Stretching & 10 & $1-12$ \\
\hline \multirow{5}{*}{ J } & &
\end{tabular}

tor skills, the examiner awarded 1 point if they completed the task and no points if they were not able to do the task. The two results were added together for a total score using subscales. The highest possible sum of the six subscales in the locomotion section and in the object control section were 48 points, respectively.

\section{Educational exercise program}

Participants took part in a supervised educational exercise program twice a week (Tuesdays and Fridays) for 12 weeks as shown in Table 3. For the duration of the study, the participants agreed to keep their routine activity patterns the same, with the exception of their participation in the educational exercise program. The participants began with $5 \mathrm{~min}$ of warm-up led by an instructor during the 12 weeks. They then performed five types of workout sessions for 40 to $45 \mathrm{~min}$. Lastly, there was a stretching session for $10 \mathrm{~min}$.

\section{Statistical analysis}

Microsoft Excel (Microsoft, Redmond, WA, USA) was used to input the results, and the technical statistics (mean \pm standard deviation) was calculated using IBM SPSS Statistics ver. 22.0 (IBM Co., Armonk, NY, USA). The Shapiro-Wilk test was used to check the distribution of data. Prior to analysis, we observed the difference between groups through Mann-Whitney $U$-test, as shown in Table 1. Then, the effects of the educational exercise program
Table 4. Comparative results of body composition

\begin{tabular}{|c|c|c|c|c|c|c|}
\hline \multirow[t]{2}{*}{ Item } & \multirow{2}{*}{$\begin{array}{l}\text { Time } \\
\text { (T) }\end{array}$} & \multicolumn{2}{|c|}{ Group (G) } & \multicolumn{3}{|c|}{$\begin{array}{c}\text { Repeated ANOVA } \\
(P \text {-value })\end{array}$} \\
\hline & & Mild ASD $(n=17)$ & Severe ASD $(n=18)$ & G & $\mathrm{T}$ & $G^{*} T$ \\
\hline \multirow{2}{*}{$\begin{array}{l}\text { Body weight } \\
(\mathrm{kg})\end{array}$} & Pre & $73.02 \pm 12.63$ & $72.09 \pm 10.32$ & 0.842 & 0.245 & 0.001 \\
\hline & Post & $70.87 \pm 10.05$ & $73.26 \pm 10.14$ & & & \\
\hline \multirow{2}{*}{$\begin{array}{l}\text { BMl } \\
\qquad\left(\mathrm{kg} / \mathrm{m}^{2}\right)\end{array}$} & Pre & $24.18 \pm 4.32$ & $24.14 \pm 4.18$ & 0.707 & 0.253 & 0.001 \\
\hline & Post & $23.47 \pm 3.50$ & $24.53 \pm 4.14$ & & & \\
\hline \multirow{2}{*}{$\begin{array}{l}\text { Muscle } \\
\text { mass (kg) }\end{array}$} & Pre & $51.47 \pm 6.97$ & $51.61 \pm 6.62$ & 0.920 & 0.005 & 0.013 \\
\hline & Post & $52.26 \pm 7.12$ & $51.66 \pm 6.56$ & & & \\
\hline \multirow{2}{*}{$\begin{array}{l}\text { Fat mass } \\
(\mathrm{kg})\end{array}$} & Pre & $21.75 \pm 8.20$ & $24.14 \pm 6.99$ & 0.209 & 0.227 & 0.001 \\
\hline & Post & $21.24 \pm 7.25$ & $25.09 \pm 6.40$ & & & \\
\hline \multirow{2}{*}{$\begin{array}{l}\text { BMR } \\
\text { (kcal/day) }\end{array}$} & Pre & $1,596.82 \pm 120.19$ & $1,561.67 \pm 162.84$ & 0.278 & 0.523 & 0.070 \\
\hline & Post & $1,608.00 \pm 131.06$ & $1,538.72 \pm 150.06$ & & & \\
\hline
\end{tabular}

Values are presented as mean \pm standard deviation.

ANOVA, analysis of variance; ASD, autism spectrum disorder; BMl, body mass index; BMR, basal metabolic rate.

were evaluated using repeated $2 \times 2$ analysis of variance (group, time, and group by time interaction). The intergroup factor was the study groups (i.e., mild ASD group vs. severe ASD group) and the intragroup factor was the time (i.e., baseline vs. week 12). $\Delta \%$ was calculated with further analysis of the variables between times. For all analyses, a significance level of $P \leq 0.05$ was set.

\section{RESULTS}

\section{Comparison of demographic factors}

A total of 35 male adults voluntarily participated in this study (Table 1). All participants were 20 to 29 years of age, with a mean of $22.83 \pm 2.72$ years.

\section{Differences in body composition depending on severity of ASD}

As shown in Table 4, the participants showed similar results, except for BMR. In detail, although the $\Delta \%$ in body weight and BMI in mild ASD group decreased $~ 2.34 \%$, those of severe ASD group increased $\sim 1.70 \%$ (not shown in the table). In the aspect of the $\Delta \%$ in muscle mass, mild ASD group increased $\sim 1.54 \%$, while that of severe ASD group increased $\sim 0.13 \%$. Although the $\Delta \%$ in fat mass in mild ASD group decreased $\sim 0.28 \%$, that of severe ASD group increased $\sim 5.60 \%$. The $\Delta \%$ in BMR in mild ASD group increased $\sim 0.75 \%$, while that of severe ASD group decreased $\sim 1.39 \%$ after 12 weeks. This indicates that the educational exercise program provided a greater positive effect for those with mild ASD. These results show a significant difference in interaction, except for BMR. 
Table 5. Comparative results of physical fitness

\begin{tabular}{|c|c|c|c|c|c|c|}
\hline \multirow{2}{*}{ Item } & \multirow{2}{*}{$\begin{array}{c}\text { Time } \\
(T)\end{array}$} & \multicolumn{2}{|c|}{ Group (G) } & \multicolumn{3}{|c|}{$\begin{array}{l}\text { Repeated ANOVA } \\
\text { ( } P \text {-value })\end{array}$} \\
\hline & & $\begin{array}{l}\text { Mild ASD } \\
(n=17)\end{array}$ & $\begin{array}{c}\text { Severe ASD } \\
(n=18)\end{array}$ & G & $\mathrm{T}$ & $\mathrm{G}^{*} \mathrm{~T}$ \\
\hline \multirow{2}{*}{$\begin{array}{r}\text { Cardiopulmonary } \\
\text { endurance (sec) }\end{array}$} & Pre & $6.09 \pm 0.33$ & $6.13 \pm 0.31$ & 0.006 & 0.007 & 0.001 \\
\hline & Post & $6.59 \pm 0.35$ & $5.95 \pm 0.40$ & & & \\
\hline \multirow[t]{2}{*}{ Flexibility (cm) } & Pre & $-6.21 \pm 3.89$ & $-6.33 \pm 3.34$ & 0.001 & 0.001 & 0.001 \\
\hline & Post & $-0.54 \pm 2.99$ & $-7.68 \pm 2.80$ & & & \\
\hline \multirow[t]{2}{*}{ Strength (kg) } & Pre & $17.19 \pm 4.59$ & $15.78 \pm 5.10$ & 0.264 & 0.001 & 0.732 \\
\hline & Post & $19.44 \pm 4.23$ & $17.66 \pm 3.79$ & & & \\
\hline \multirow[t]{2}{*}{ Endurance (rep.) } & Pre & $14.29 \pm 2.52$ & $13.28 \pm 2.11$ & 0.596 & 0.001 & 0.001 \\
\hline & Post & $15.76 \pm 2.54$ & $11.44 \pm 1.79$ & & & \\
\hline
\end{tabular}

Values are presented as mean \pm standard deviation.

ANOVA, analysis of variance; $A S D$, autism spectrum disorder.

\section{Differences in physical fitness depending on levels of ASD}

As shown in Table 5, the participants showed similar results, except for strength. Specifically, although the $\Delta \%$ in cardiopulmonary endurance in mild ASD group increased $\sim 8.49 \%$, that of severe ASD group decreased $\sim 2.88 \%$ (not shown in the table). In the aspect of the $\Delta \%$ in flexibility, mild ASD group improved $\sim 91.3 \%$, while that of severe ASD group reduced $\sim 21.3 \%$. The $\Delta \%$ in strength in mild ASD group increased $\sim 16.92 \%$, and that of severe ASD group also showed a similar increase of $\sim 17.20 \%$. The $\Delta \%$ in muscle endurance in mild ASD group increased $\sim 11.34 \%$, while that of severe ASD group decreased $~ 12.48 \%$ after 12 weeks. This indicates that the educational exercise program provided a positive effect for participants with mild ASD, which was confirmed by a significant difference in interaction, except for muscle strength.

\section{Differences in GMF depending on levels of ASD}

As shown in Table 6, there were significant differences in the interaction of all variables, except for the locomotion skill of hopping. However, there were significant differences in the interaction of all variables of object control skill. Specifically, although the $\Delta \%$ in the sum of locomotion skill in mild ASD group had a baseline of $32.76 \pm 5.91$, there was an insignificant increase to $38.71 \pm 4.67$ $(\sim 19.81 \%)$ after the experiment. However, that of severe ASD group significantly decreased from $32.61 \pm 6.82$ before the experiment, to $30.50 \pm 4.23(\sim 4.78 \%)$ after 12 weeks (interaction; $P=$ 0.001) (Fig. 1. left). The $\Delta \%$ in the sum of object control skill in mild ASD group with a baseline of 23.24 44.92 showed an insignificant increase to $29.06 \pm 3.15(\sim 29.96 \%)$ after the experiment. However, that of severe ASD group significantly decreased from
Table 6. Comparative results of gross motor function

\begin{tabular}{|c|c|c|c|c|c|c|}
\hline \multirow{2}{*}{ Item } & \multirow{2}{*}{$\begin{array}{l}\text { Time } \\
(\mathrm{T})\end{array}$} & \multicolumn{2}{|c|}{ Group (G) } & \multicolumn{3}{|c|}{$\begin{array}{l}\text { Repeated ANOVA } \\
\quad(P \text {-value })\end{array}$} \\
\hline & & $\begin{array}{l}\text { Mild ASD } \\
\quad(n=17)\end{array}$ & $\begin{array}{l}\text { Severe ASD } \\
\quad(n=18)\end{array}$ & G & $\mathrm{T}$ & $G^{*} T$ \\
\hline \multicolumn{7}{|l|}{ Locomotion skill } \\
\hline \multirow{2}{*}{ Running } & Pre & $4.71 \pm 2.02$ & $4.78 \pm 2.60$ & 0.347 & 0.005 & 0.008 \\
\hline & Post & $6.12 \pm 1.36$ & $4.83 \pm 1.82$ & & & \\
\hline \multirow[t]{2}{*}{ Galloping } & Pre & $6.35 \pm 1.27$ & $6.50 \pm 1.38$ & 0.001 & 0.358 & 0.001 \\
\hline & Post & $7.12 \pm 0.86$ & $5.33 \pm 0.91$ & & & \\
\hline \multirow[t]{2}{*}{ Hopping } & Pre & $5.88 \pm 1.36$ & $6.06 \pm 1.63$ & 0.640 & 0.006 & 0.081 \\
\hline & Post & $6.82 \pm 1.13$ & $6.28 \pm 1.02$ & & & \\
\hline \multirow[t]{2}{*}{ Leaping } & Pre & $5.47 \pm 0.72$ & $5.83 \pm 0.79$ & 0.123 & 0.011 & 0.001 \\
\hline & Post & $6.65 \pm 1.32$ & $5.44 \pm 0.62$ & & & \\
\hline \multirow{2}{*}{$\begin{array}{l}\text { Horizontal } \\
\text { jumping }\end{array}$} & Pre & $3.29 \pm 2.85$ & $2.39 \pm 2.66$ & 0.047 & 0.081 & 0.006 \\
\hline & Post & $4.47 \pm 2.43$ & $2.11 \pm 1.75$ & & & \\
\hline \multirow[t]{2}{*}{ Sliding } & Pre & $7.06 \pm 1.09$ & $7.06 \pm 1.00$ & 0.111 & 0.808 & 0.006 \\
\hline & Post & $7.53 \pm 1.23$ & $6.50 \pm 0.92$ & & & \\
\hline \multicolumn{7}{|c|}{ Object (ball) control skill } \\
\hline \multirow[t]{2}{*}{ Striking } & Pre & $3.59 \pm 1.00$ & $3.39 \pm 1.38$ & 0.004 & 0.163 & 0.002 \\
\hline & Post & $4.71 \pm 0.99$ & $2.94 \pm 1.21$ & & & \\
\hline \multirow[t]{2}{*}{ Dribbling } & Pre & $6.06 \pm 2.30$ & $7.06 \pm 1.59$ & 0.870 & 0.318 & 0.005 \\
\hline & Post & $7.29 \pm 0.85$ & $6.44 \pm 1.34$ & & & \\
\hline \multirow[t]{2}{*}{ Catching } & Pre & $4.88 \pm 1.36$ & $4.89 \pm 1.13$ & 0.005 & 0.390 & 0.001 \\
\hline & Post & $5.76 \pm 0.90$ & $3.61 \pm 1.54$ & & & \\
\hline \multirow[t]{2}{*}{ Kicking } & Pre & $5.12 \pm 1.54$ & $5.61 \pm 1.42$ & 0.111 & 0.903 & 0.001 \\
\hline & Post & $6.24 \pm 1.20$ & $4.44 \pm 1.04$ & & & \\
\hline \multirow[t]{2}{*}{ Overthrowing } & Pre & $0.12 \pm 0.33$ & $0.06 \pm 0.24$ & 0.013 & 0.001 & 0.016 \\
\hline & Post & $0.76 \pm 0.66$ & $0.22 \pm 0.43$ & & & \\
\hline \multirow[t]{2}{*}{ Under rolling } & Pre & $3.47 \pm 1.77$ & $3.11 \pm 1.64$ & 0.030 & 0.704 & 0.001 \\
\hline & Post & $4.29 \pm 1.65$ & $2.44 \pm 1.15$ & & & \\
\hline
\end{tabular}

Values are presented as mean \pm standard deviation.

ANOVA, analysis of variance; ASD, autism spectrum disorder.

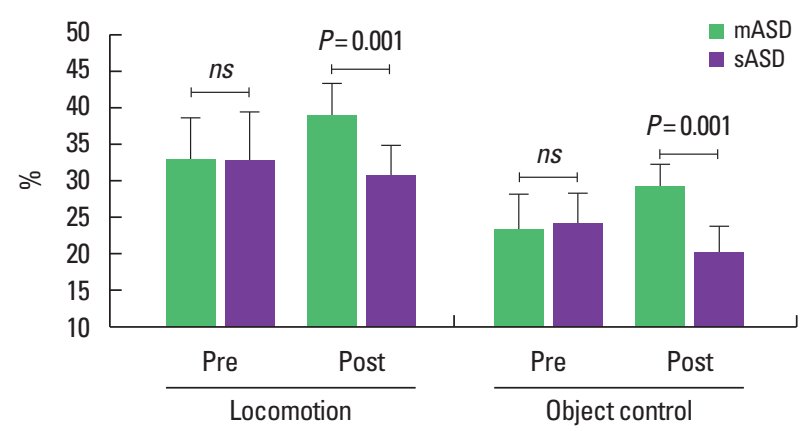

Fig. 1. Differences of the $\Delta \%$ of the sum of locomotion and object control skills of gross motor function in mild autism spectrum disorder group (mASD) and severe autism spectrum disorder group (SASD) between baseline and 12 weeks. ns, nonsignificant.

$24.11 \pm 4.24$ before the experiment, to $20.11 \pm 3.53(\sim 15.2 \%)$ after 12 weeks (interaction; $P=0.001$ ) (Fig. 1. right). 


\section{DISCUSSION}

Most of the educational exercise programs related to ASD have only focused on children. The adult population, as well as the severity of ASD, were not taken into consideration by such intervention programs. Therefore, this study aimed to investigate physical fitness and GMF in adult participants with mild or severe ASD who took part in a 12-week educational exercise program. Upon completion of the experiment, this study found significant differences in the interaction of body composition and physical fitness variables, except muscle strength, when comparing between mild and severe ASD groups. This study also found that there were significant differences in the locomotion skill and object control skill of GMF between groups. These results suggest that individuals with more severe levels of ASD may have more difficulty in adapting to and understanding the educational exercise program while those with milder levels of ASD may have an easier time adapting to and understanding the program.

Many researchers have reported that adults with ASD are characterized by decreased physical, cognitive, and adaptive skills when compared to those without ASD. Moreover, they are often stereotyped with having challenging behaviors (Downs et al., 2008; Holden and Gitlesen, 2008; Lee et al., 2008; Lifshitz et al., 2008; Matson et al., 2005; McGillivray et al., 2008; Myrbakk and von Tetzchner, 2008; Yalon-Chamovitz and Weiss, 2008; Zayac and Johnston, 2008). Rapp et al. (2004) reported on the possibility that the physical stimulation obtained via physical activity may be similar to certain stereotypic behaviors such as body rocking, arm flapping, and spinning in circles. Such behaviors are often hypothesized to occur because the behavior itself produces pleasant internal consequences for the individual with disability. Since a physical activity may involve similar body mechanics to that of stereotypical behavior, it may produce similar internal states, making it possible that the participants' need for this automatic reinforcement is obtained sufficiently during the activity sessions (Lang et al., 2010). Ortega et al. (2015) suggested that physical fitness levels have long-term effects and indicate a person's overall health. Bürgi et al. (2011) indicated that the level of physical activity is associated with improvements in aerobic capacity. However, unlike previous studies (Lang et al., 2010; Ortega et al., 2015), this study revealed that physical activity does not equally provide positive changes to all people with disability. That is, physical activity can provide different results depending on the degree of disability. From the results of this study, cardiopulmonary endurance, that is, aerobic capacity, had varying differences according to the degree of ASD. Similarly, most of the physical fitness variables (flexibility and muscle endurance) changed positively in mild ASD group compared to severe ASD group after 12 weeks. These results indicate that the educational exercise program provided a greater positive effect for those with mild ASD, which was confirmed by a significant difference in interaction after 12 weeks.

However, there was no significant difference in muscle strength between the two groups. This may be due to the fact that the educational exercise program mainly focused on developing endurance. The effect of fatigue is a factor that may influence such a result. A study by Rosenthal-Malek and Mitchell (1997) trained five child participants with ASD to jog 20 minutes. After the jog, self-stimulatory behavior in the community work place and classroom decreased substantially, which could possibly be explained by fatigue. However, responses to academic demands and work tasks completed increased after educational play and/or exercise. This suggests that participants were not overly fatigued to work with improved proficiency and accuracy. In other words, muscle strength is a variable opposite to fatigue, and this study revealed that even if educational exercise programs are provided, it may affect individuals differently depending on the severity of their disability. Previous studies have shown numerous cases of people with disabilities who have a high level of physical fitness. Physical activity provided to adults with mental retardation has been reported to promote the physical health of people with disabilities (Jansen et al., 2004). This study revealed that the body composition in adults with ASD was positively changed by the end of the experiment. Specifically, the results of this study showed a greater positive effect in mild ASD group than severe ASD group. This context was in line with the physical fitness variables suggested above. Physical activity also enhances the ability of the large and small muscles to function and protect the body for people with developmental disabilities (Verschuren et al., 2016). Similar to these previous studies, this study showed improvement in most of the variables for locomotion skill and object (ball) control skill. However, the GMFs of mild ASD group were significantly different between both groups as well. Specifically, the sum of locomotion skill in mild ASD group increased $\sim 19.81 \%$, while that of severe ASD group decreased $\sim 4.78 \%$. The sum of object control skill in mild ASD group improved $29.96 \%$, while that of severe ASD group decreased $\sim 15.2 \%$. In other words, it can be said that the educational exercise program applied in this study led to different results depending on the severity of disability.

Gabler-Halle et al. (1993) suggested that the appeal and application of physical fitness intervention programs for people with 
developmental disabilities would be greater if it were placed in the context of positive behavioral and psychological change. The findings from this study suggest that exercise not only leads to improved physical fitness, but also to positive changes in behavior. However, the mechanism of action for these improvements is unclear. In some studies, there was limited information regarding the methods used to instruct exercises to individuals with ASD. However, activities such as jogging, may not require extensive procedures for teaching. Indeed, several common procedures and strategies emerged across studies (Cha et al., 2020). First, embedding exercise into age-appropriate activities and games may increase motivation to engage in exercise, similar to the program used in this study. If exercise becomes a reinforcing or preferable activity, procedures for increasing exercise may be simplified, which would require less programmed reinforcement. Second, physical prompts gradual fading was the most common approach in most studies. Finally, reinforcement through social praise may be required to encourage participation in exercise, especially when the exercise is not a preferred activity.

To date, exercise programs for individuals with ASD have only been implemented in an educational environment, and not according to gender, age, or severity of disability. However, this study showed that adults with ASD who participated in the educational exercise program had differing results based on the severity of their disability. It would beneficial for future studies to investigate the effects of exercise on other types of disorders. Also, there is a need to research the mechanisms on how exercise positively influences behaviors. Gaining an understanding of this mechanism may lead to improvements in applying exercise to treat various levels of ASD and help practitioners develop more effective programs for people with ASD.

This study found that educational exercise programs have a positive effect on adults with ASD. However, it affects adults with ASD according to the severity of their disability. In other words, this study revealed that adults with ASD who participated in the educational exercise program showed differences in physical fitness and GMF according to the severity of their ASD. In conclusion, these results are thought to be due to the better understanding and performance of educational exercise in adults with mild ASD compared to severe ASD.

\section{CONFLICT OF INTEREST}

No potential conflict of interest relevant to this article was reported.

\section{REFERENCES}

Ahn S, Fedewa AL. A meta-analysis of the relationship between children's physical activity and mental health. J Pediatr Psychol 2011;107:1-13.

Baird G, Simonoff E, Pickles A, Chandler S, Loucas T, Meldrum D, Charman T. Prevalence of disorders of the autism spectrum in a population cohort of children in South Thames: the Special Needs and Autism Project (SNAP). Lancet 2006;368:210-215.

Barbotte E, Guillemin F, Chau N. Prevalence of impairments, disabilities, handicaps and quality of life in the general population: a review of recent literature. Bull World Health Organ 2001;79:1047-1055.

Brugha TS, McManus S, Bankart J, Scott F, Purdon S, Smith J, Bebbington $\mathrm{P}$, Jenkins R, Meltzer H. The epidemiology of autism spectrum disorders in adults in the community in England. Arch Gen Psychiatry 2011; 68:459-466.

Bürgi F, Meyer U, Granacher U, Schindler C, Marques-Vidal P, Kriemler S, Puder JJ. Relationship of physical activity with motor skills, aerobic fitness and body fat in preschool children: a cross-sectional and longitudinal study (Ballabeina). Int J Obesity 2011;35:937-944.

Cha JY, Kim JH, Hong J, Choi YT, Kim MH, Cho JH, Ko IG, Jee YS. A 12week rehabilitation program improves body composition, pain sensation, and internal/external torques of baseball pitchers with shoulder impingement symptom. J Exerc Rehabil 2014;10:35-44.

Cha JY, Min SK, Yoon TH, Jee YS. Gross motor function and health fitness in adults with autistic spectrum disorder and intellectual disability: single-blind retrospective trial. J Exerc Rehabil 2020;16:258-264.

Downs A, Downs RC, Rau K. Effects of training and feedback on discrete trial teaching skills and student performance. Res Dev Disabil 2008;29: 235-246.

Gabler-Halle D, Halle JW, Chung YB. The effects of aerobic exercise on psychological and behavioral variables of individuals with developmental disabilities: a critical review. Res Dev Disabil 1993;14:359-386.

Holden B, Gitlesen JP. The relationship between psychiatric symptomatology and motivation of challenging behaviour: a preliminary study. Res Dev Disabil 2008;29:408-413.

Howlin P, Moss P. Adults with autism spectrum disorders. Can J Psychiatry 2012;57:275-283.

Jansen DE, Krol B, Groothoff JW, Post D. People with intellectual disability and their health problems: a review of comparative studies. J Intellect Disabil Res 2004;48:93-102.

Janssen I, LeBlanc AG. Review systematic review of the health benefits of physical activity and fitness in school-aged children and youth. Int J Behav Nutr Phy 2010;7:1-16.

Kim S, Jee Y. Effects of 3D moving platform exercise on physiological parameters and pain in patients with chronic low back pain. Medicina 
(Kaunas) 2020;56:351.

Lang R, Koegel LK, Ashbaugh K, Regester A, Ence W, and Smith W. Physical exercise and individuals with autism spectrum disorders: a systematic review. Res Autism Spectr Disord 2010;4:565-576.

Latorre Román PÁ, Mora López D, Fernández Sánchez M, Salas Sánchez J, Moriana Coronas F, García-Pinillos F. Test-retest reliability of a fieldbased physical fitness assessment for children aged 3-6 years. Nutr Hosp 2015;32:1683-1688.

Lee LC, Harrington RA, Chang JJ, Conners SL. Increased risk of injury in children with developmental disabilities. Res Dev Disabil 2008;29:247255.

Lifshitz H, Merrick J, Morad M. Health status and ADL functioning of older persons with intellectual disability: community residence versus residential care centers. Res Dev Disabil 2008;29:301-315.

Matson JL, Dixon DR, Matson ML, Logan JR. Classifying mental retardation and specific strengths and deficit areas in severe and profound mentally retarded persons with the MESSIER. Res Dev Disabil 2005; 26:41-45.

McGillivray JA, McCabe MP, Kershaw MM. Depression in people with intellectual disability: an evaluation of a staff-administered treatment program. Res Dev Disabil 2008;29:524-536.

Myrbakk E, von Tetzchner S. Psychiatric disorders and behavior problems in people with intellectual disability. Res Dev Disabil 2008;29:316-332.

Oppewal A, Hilgenkamp TI, van Wijck R, Evenhuis HM. The effect of handedness on grip strength in older adults with intellectual disabili- ties. Res Dev Disabil 2013;34:1623-1629.

Ortega FB, Cadenas-Sánchez C, Sánchez-Delgado G, Mora-González J, Martínez-Téllez B, Artero EG, Castro-Piñero J, Labayen I, Chillón P, Löf M, Ruiz JR. Systematic review and proposal of a field-based physical fitness-test battery in preschool children: the PREFIT battery. Sports Med 2015;45:533-555.

Rapp JT, Vollmer TR, St Peter C, Dozier CL, Cotnoir NM. Analysis of response allocation in individuals with multiple forms of stereotyped behavior. J Appl Behav Anal 2004;37:481-501.

Rosenthal-Malek A, Mitchell S. Brief report: the effects of exercise on the self-stimulatory behaviors and positive responding of adolescents with autism. J Autism Dev Disord 1997;27:193-202.

Verschuren O, Peterson MD, Balemans ACJ, Hurvitz EA. Exercise and physical activity recommendations for people with cerebral palsy. Dev Med Child Neurol 2016;58:798-808.

Yalon-Chamovitz S, Weiss PL. Virtual reality as a leisure activity for young adults with physical and intellectual disabilities. Res Dev Disabil 2008; 29:273-287.

Yoon TH, Mun YK, Lee JS, Min SK, Jee YS. Analysis for reliability and validity of gross motor function and health fitness tests for children with developmental disabilities. J Exerc Rehabil 2019;15:667-675.

Zayac RM, Johnston JM. Contriving establishing operations: responses of individuals with developmental disabilities during a learning task. Res Dev Disabil 2008;29:202-216. 